Revista internacional de fronteras, territorios y regiones / International Journal of Borders, Territories and Regions

FRONTERA NORTE VOL. 33, ART. 18, 2021

https://doi.org/10.33679/rfn.v1i1.2166

\title{
Nuevas geografías vitivinícolas en la frontera México-Estados Unidos. Un estudio sobre el sentido del lugar
}

New Wine Geographies on the United States-Mexico Border. A Study About the Sense of Place

María del Carmen Salas Quesada ${ }^{1}$ y Sergio Alfonso Sandoval Godoy ${ }^{2}$

\section{RESUMEN}

Este artículo aborda los casos de Sonoita-Elgin, Arizona, y Cananea, Sonora, como nuevas geografías vitivinícolas. Con el propósito de caracterizar dichas regiones y evidenciar sus potencialidades, sus problemáticas y sus retos, se privilegia el estudio socioantropológico, el método etnográfico y el análisis del sentido del lugar. Siguiendo esta línea, los hallazgos iniciales muestran primero que el sentido del lugar no viene dado por una geografía, sino por un conjunto de interacciones específicas, de donde se deriva el carácter particular de cada región; y segundo, que el contexto fronterizo ofrece una oportunidad de colaboración binacional aun por explorar, que puede traducirse en ventajas competitivas y en construcciones alternativas e innovadoras sobre el sentido del lugar. Por su alcance interpretativo, y por estar enfocado en zonas de producción emergentes, se espera contribuir a nuevos debates sobre la industria vitivinícola a nivel global y, en específico, en la región Sonora-Arizona, en la frontera México-E.E. U.U.

Palabras clave: 1. vitivinicultura, 2. nuevas geografías, 3. sentido del lugar, 4. Cananea, 5. Sonoita.

\section{ABSTRACT}

This article examines the cases of Sonoita-Elgin, Arizona, and Cananea, Sonora, as new wine geographies. To characterize these regions and show their potential, problems, and challenges, this article privileges the socio-anthropological study, the ethnographic method, and the analysis of the sense of place. The initial findings show first that the sense of place is not given by geography but by a set of specific interactions that conform the particular character of each region; and second, that the border context offers an opportunity for binational collaboration yet to be explored, which can be translated into competitive advantages and into alternative and innovative constructions of the sense of place. Due to the interpretative extent of this analysis and its focus on emerging production areas, it is expected to contribute to new discussions on the wine industry globally and, specifically, on the region of Sonora-Arizona in the U.S.-Mexico border region.

Keywords: 1. viticulture, 2. new geographies, 3. sense of place, 4. Cananea, 5. Sonoita.

Fecha de recepción: 21 de septiembre de 2020

Fecha de aceptación: 4 de diciembre de 2020

Publicación web: 30 de septiembre de 2021

\footnotetext{
${ }^{1}$ Centro de Investigación en Alimentación y Desarrollo, México, carmensalasquesada@gmail.com, https://orcid.org/0000-0002-3456-9778.

${ }^{2}$ Centro de Investigación en Alimentación y Desarrollo, México. Autor de correspondencia, ssandoval@ciad.mx, https://orcid.org/0000-0002-8380-9520.
} 
2 Nuevas geografías vitivinícolas en la frontera México-Estados Unidos. Un estudio sobre el sentido... Salas Quesada, M.C. y Sandoval Godoy, S.G.

\section{INTRODUCCIÓN ${ }^{3}$}

En el siglo XXI la industria del vino se encuentra en una nueva fase de producción y consumo, en la que uno de los fenómenos característicos corresponde a la expansión y diversificación de la vitivinicultura hacia regiones y localidades consideradas como "no tradicionales". Lo anterior ha provocado respuestas locales muy diversas, en la medida que se cuestionan o se reinterpretan ideas como el terroir (terruño), el saber hacer, la autenticidad o las denominaciones de origen, entre otros aspectos. De manera que la expansión de la industria del vino no sólo ha ocasionado nuevas relaciones e interpretaciones sobre las cualidades del producto y su lugar de origen, sino también ha evidenciado los dilemas de la globalización, del desarrollo económico-político, y de la evolución de las prácticas y los significados culturales asociados al valor y a la producción de actividades como la vitivinicultura (Demossier, 2013, 2018; Harvey, 2002).

Los proyectos de producción de vino surgidos en la década de los ochenta en Sonoita, Arizona, Estados Unidos, y al finalizar la década de los dosmil en Cananea, Sonora, México, son ejemplos representativos de estas dinámicas productivas de cambio, ligadas al surgimiento de las llamadas "nuevas geografías" vitivinícolas, o "áreas vitivinícolas emergentes o en desarrollo" (Banks y Overton, 2010). Estas nuevas geografias vitivinícolas (NGV) de la frontera México-Estados Unidos inauguran una función productiva completamente diferente al resto de actividades de la zona donde se localizan. Aparecen como una mezcla de influencias creativas de experimentación, adaptación e innovación, de ideas tradicionales y conservadoras, y con distintas potencialidades económicas, culturales y de impacto social. No obstante, también traen consigo problemáticas complejas que podrían estar inhibiendo sus expectativas de éxito y su impacto en el desarrollo local. Tal como lo señalan varios autores, algunas de estas problemáticas están relacionadas con el papel que desempeña el territorio (Moran, 1993); entendido este en un sentido antropológico como un "ambiente de vida, de acción, y de pensamiento de una comunidad, asociado a procesos de construcción de identidad" (Tizon, 1995, citado en Flores, 2007, p. 36); pero también con el rol que juegan la población local y la identidad regional. Dichas problemáticas se pueden analizar desde lo que se ha dado en llamar "la producción del lugar" (Appadurai, 1986, 1996; Escobar, 2001; Friedman,

3 Este artículo es parte del proyecto de investigación 39562 denominado $L a$ reconfiguración y nuevas funciones de Las fronteras del siglo XXI: entre la integración/desintegración, desfronterización/refronterización y cooperación/conflicto, financiado por el Conacyt a través de la convocatoria Fronteras de la Ciencia (Fondo Institucional de Fomento Regional para el Desarrollo Científico, Tecnológico y de Innovación/Programas Nacionales Estratégicos). Asimismo, la presente investigación doctoral de la que forma parte este trabajo y que se centra en el análisis sobre las nuevas geografías vitivinícolas y la creación de valor, cuenta desde enero de 2021 y hasta enero de 2022 con el apoyo adicional de una beca de investigación por parte de la Organización Internacional de la Viña y el Vino (OIV). 
1994; Ingold, 1993; Urry, 1995), the making (Demossier, 2018; Ferguson, 1998; Rainer, 2016), la puesta en valor (Giménez, 1997, 1999), el sentido del lugar (Demossier, 2018; Massey, 1991; Paxson, 2010; Trubek y Bowen, 2008), o the embeddedness (Bowen, 2010; Murdoch, Marsden y Banks, 2000; Winter, 2003).

El razonamiento detrás de estas conjeturas aplicadas al estudio de la producción vitivinícola es que, por tratarse de una mercancía no estandarizada (Appadurai, 1986), el vino depende de ciertas condiciones que le confieren características únicas como producto y como actividad. Su manejo mercantil y su conexión con el lugar de origen le dan un valor material y simbólico difícil de encontrar en otros bienes. De este modo, aunque se trata de un producto y una actividad económica que se reproduce y expande a nivel global, sus especificidades regionales o locales conllevan marcadas diferencias.

Con el propósito de generar información novedosa, privilegiamos el análisis del sentido del lugar como uno de los procesos y estrategias que intervienen en la construcción de los modelos de producción, distribución y consumo; así como para examinar la relación con la población local y el desarrollo de la industria y la región. Siguiendo esta línea analítica y utilizando el método etnográfico, comparamos las trayectorias de los nuevos asentamientos vitivinícolas de Sonoita-Elgin, en Arizona, E.E. U.U., y Cananea, en Sonora, México, para probar que se trata de experiencias de impacto positivo en la región desde las que podemos explicar y contrastar los avances y los desafíos que se presentan a la hora de introducir la vitivinicultura como alternativa productiva, como motor para el desarrollo y como iniciativa para la interacción de dichos espacios fronterizos. Por tratarse de una zona de producción reciente y poco estudiada, este trabajo representa un primer acercamiento al estudio de dicha región fronteriza productora de vino desde el análisis socioantropológico de la producción del lugar.

Conscientes de que la complejidad de dicho fenómeno requiere de análisis relacionales e integrales todavía más profundos, enseguida desarrollamos cuatro apartados en los que primeramente se exponen las implicaciones teóricas del análisis del sentido del lugar y se presentan algunas experiencias relacionadas con dicho proceso; después se explican las características de las NGV como unidad general de análisis y se presenta el diseño del trabajo de campo; posteriormente se analizan las particularidades y hallazgos preliminares que conllevan los casos de Sonoita-Elgin y Cananea; finalmente, se exponen las conclusiones centrales.

\section{LA VOZ DEL POTENCIAL VITIVINÍCOLA A TRAVÉS DEL SENTIDO DEL LUGAR Y LA IDENTIDAD PRODUCTIVA}

El sentido del lugar es un concepto antiguo que en geografía se ha utilizado para comprender la relación espacio-tiempo, y para analizar cómo a partir de esta relación cambiante se configura la especificidad y/o la identidad en el contexto de cada sociedad (Massey, 1991). Así, el sentido del lugar se puede analizar como una versión de la(s) identidad(es) que deriva de su "función locativa" (Giménez, 1997, p. 23). 
4 Nuevas geografías vitivinícolas en la frontera México-Estados Unidos. Un estudio sobre el sentido... Salas Quesada, M.C. y Sandoval Godoy, S.G.

De acuerdo con Massey (1991), dicho concepto a menudo se ha interpretado de manera errónea como una forma de identidad ligada al pasado, fija: "como una respuesta al deseo de seguridad y estabilidad en un momento en el que todo es movimiento y es cambio" (Massey, 1991, p. 26 [traducción propia]); como un recurso ante la aparente pérdida de control, de desterritorialización derivada del proceso de globalización. Sin embargo, como indica la autora, la anterior interpretación es inadecuada por las siguientes razones: en primer lugar, porque no se ajusta al presente globalizado (Haesbaert, 2011) en el que vivimos. Es decir, que el sentido del lugar no se construye mirando solo hacia atrás y hacia dentro, sino también hacia afuera y al futuro, y donde lo global y lo local se combinan de manera simultánea configurando "un nuevo proceso" (Haesbaert, 2011, p. 287). En segundo lugar, porque para cada espacio no existe una sola y estática identidad, sino que son múltiples y cambiantes. $\mathrm{Y}$ en tercer lugar, porque de manera igualmente errónea se tiene la idea de que el sentido del lugar implica comunidad, homogeneidad y ausencia de conflictos. Al contrario, se advierte que la construcción del lugar conlleva unos límites, unas fronteras, un nosotros frente a ellos, así como un juego de poderes entre individuos y grupos con diferentes posiciones en la sociedad. Al reparar en estos aspectos, la red de interacciones que van a caracterizar la producción del lugar se complejiza, y por tanto, se alejan de ese imaginario romántico del sentido del lugar como un espacio homogéneo y sin conflictos.

Las ideas anteriores son fundamentales para el análisis sobre las NGV al subrayar que la especificidad de un lugar, de la cual depende el valor (cualitativo y cuantitativo) de una región vitivinícola, va a depender no solo de una condición geográfica delimitada (Moran, 1993), sino también de una construcción de relaciones socioculturales a diferentes escalas:

[...] Lo que le da a un lugar su especificidad no es una larga historia interiorizada, sino el hecho de que esta se construye a partir de una constelación particular de relaciones sociales que se encuentran y se tejen en un lugar particular [...] Y esto a su vez permite que se exprese un sentido del lugar en el que se incluye una conciencia de sus vínculos con el mundo más amplio, el cual integra de manera positiva lo global y lo local (Massey, 1991, p. 28 [traducción propia]).

La producción de vino y la estrategia de valorización y diferenciación con base en el sentido del lugar, se configuran de manera compleja y dejan ver que para desarrollar una alternativa de estas características, identificar el potencial es solo el primer paso. Como expone Appadurai (1996, pp. 180-181), el lugar, su potencialidad y su especificidad es volátil, es "efímera a menos que se emprenda un trabajo duro para producir y mantener su materialidad" [traducción propia].

Lo anterior sugiere que es necesario construir un soporte, una estructura que permita comunicar, "dar voz" (Raftery, 2017) a esa potencialidad; esto es, en el sentido de materializar, representar y comunicar la singularidad de un área vitivinícola determinada. De manera convencional, este proceso se ha traducido y materializado en un sistema de indicaciones geográficas y/o diferentes versiones de una identidad productiva que puede 
configurarse de manera más instrumental, como el place marketing, o más simbólica, como el discurso del terroir (Barham, 2003; Demossier, 2013). Estrategias que a su vez se hibridan y se complementan a distintos niveles para cada caso:

El terroir es una especie de place marketing [...] pero uno que no crea simplemente una asociación superficial con un lugar a través de un producto para generar ventas. En cambio, refleja un esfuerzo concreto para crear literalmente las bases sociales y económicas con las que revindicar la singularidad y la reputación de productos de calidad o de alto valor agregado (Barham, 2007, p. 279; citado en Paxson, 2010, p. 452 [traducción propia]).

En este trabajo, la identidad productiva se entiende, para el caso de la industria vitivinícola y/o gastronómica, como el resultado de una estrategia de valorización basada en un esfuerzo colectivo de representación mediante el cual se identifican, se codifican y se comunican las relaciones entre una determinada industria o producto y los atributos específicos que le otorgan el lugar donde se produce (Contreras y Gracia, 2005; Demossier, 2018; Prats, 1997).

De acuerdo con Prats (1997), las versiones complejas y colectivas de una identidad casi siempre involucran al poder político. Dicho poder es representado a través de un aparato institucional (nacional y/o estatal o regional) que se ocupa de establecer las bases y el control de un sistema regulatorio para esta actividad, como ocurre con las denominaciones de origen. Por lo general, la población local por sí sola no tiene el poder para activar este tipo de procesos. Por su parte, el poder económico de manera aislada, en ocasiones, no es lo que busca, ya que el negocio se conforma a menudo "con construcciones simbólicas mucho más simples" (Prats, 1997, pp. 33-34).

Por lo tanto, el sentido del lugar va a resultar de la intersección de distintos tipos de relaciones entre capitales (natural, social, cultural y económico), así como de la interacción de las fuerzas globales y locales, y de las diferentes acciones llevadas a cabo por actores involucrados en cada caso (Escobar, 2001). De esa constelación de relaciones pueden surgir distintas estrategias y en consecuencia distintos resultados. Asimismo, el que se escoja una u otra composición también está condicionado por el sistema de valores hegemónicos (Bourdieu, 1998; Herzfeld, 2004; Jung, 2014) que predominen en ese momento. Esto es, por el conjunto de valores y creencias que existen en torno a la producción y el consumo de vino: desde la clasificación de las variedades en función de su reputación en el mercado, o las prácticas convencionales para la vinificación, hasta la manera de consumir distintos tipos de vino.

En definitiva, el sentido del lugar no son las características geográficas de un determinado espacio, ni tampoco un potencial por descubrir, ni una historia qué inventar. Es el resultado de un trabajo duro, como ha señalado Appadurai (1996), que pone en marcha una producción del espacio y de la cultura en el cual se expresan y se entrelazan las transformaciones de la naturaleza y la sociedad (Swyngedouw, 1999). Es a través de este proceso que se configura "la excepcionalidad cultural" (Prats, 1997, p. 23) de cada 
6 Nuevas geografías vitivinícolas en la frontera México-Estados Unidos. Un estudio sobre el sentido...

Salas Quesada, M.C. y Sandoval Godoy, S.G.

proyecto, y nos permite interpretar por qué de una geografía similar, se puedan desprender resultados muy diferentes.

\section{EXPERIENCIAS RELACIONADAS CON LA CONDICIÓN DE EL LUGAR. LA CONEXIÓN ENTRE EL PRODUCTO, LA COMUNIDAD Y EL DESARROLLO}

Buena parte de las experiencias acerca del negocio del vino a nivel mundial se ha construido bajo la premisa de que el lugar representa un factor determinante y a la vez crítico en las estrategias de rentabilidad, valorización y aceptación de ciertas marcas y regiones vitivinícolas.

En el medio académico, ha prevalecido la idea de que los modelos más exitosos con respecto al negocio del vino y la gastronomía son aquellos capaces de comunicar y convencer de manera efectiva en la existencia de una verdadera conexión entre el producto, el lugar y la comunidad (Hillel, Belhassen y Shani, 2013). Con ello se aduce la importancia de impulsar la actividad vitivinícola de manera integrada y de transmitir su especificidad y su sentido del lugar (Paxson, 2010; Trubek y Bowen, 2008; Zhao, 2005). De no cumplir esta condición, se señala que se corre el riesgo de generar un producto superficial, lo cual supone una barrera para el desarrollo y la diversificación de la industria. Distintas experiencias internacionales ponen en evidencia que "el lugar" desde el punto de vista físico es sólo uno de los elementos para este desarrollo; otros factores clave son el capital social, el territorio y la comunidad.

El trabajo de Hira y Swartz (2014) realizado en California, el de De Marchi y Grandinetti (2016) en Italia, el de Rainer (2016) para el caso de Argentina, y el de Fernández, Vaillant, Lafuente y Moreno (2017) para España, coinciden en que los aspectos que confieren mayores ventajas competitivas están relacionados con la especificidad territorial, el capital social y el emprendimiento por parte de ciertos actores estratégicos. Asimismo, en países menos conocidos en el sector del vino, el estudio de Hillel et al., (2013) llevado a cabo en Israel; el de Banks, Klinsrisuk, Dilokwanich, y Stupples (2013) en Tailandia; el de Schmidt, Macchione y Fowler de Ávila (2014) para la región vitivinícola del Vale Dos Vinhedos en Brasil; y el de Bowen (2010) para el caso de la industria tequilera en México, apuntan también otros factores importantes para la producción del lugar. Estos son el sentido de comunidad, la pertenencia territorial, el involucramiento de la sociedad, la interacción y la sinergia con la población local.

Del conjunto de experiencias se deduce que la conexión entre el producto, el lugar y la comunidad implica "poner en juego conjuntamente tres tipos de acciones: abrir la región al mundo, cultivar su especificidad histórica y cultural y, finalmente, estimular la participación de los habitantes" (Giménez, 1999, p. 52). En definitiva, se trata de vincular estos proyectos productivos y económicos con estrategias de desarrollo colectivas e integrales. 
En este sentido, la industria vitivinícola es considerada con un gran potencial para el desarrollo económico de las comunidades, y ciertamente no podemos negar el impacto positivo y la mejora en ciertos indicadores del desarrollo para algunos casos. En México, son un ejemplo Ensenada y el Valle de Guadalupe. Estos lugares han conseguido un rápido posicionamiento tanto a nivel nacional como internacional gracias a iniciativas colectivas, creativas e innovadoras como el movimiento gastronómico "BajaMed" o el programa de "ciudades gastronómicas creativas" creado por la Organización de las Naciones Unidas para la Educación, la Ciencia y la Cultura (Unesco), al que pertenece la ciudad de Ensenada desde el año 2015. ${ }^{4}$

En Baja California, la gastronomía y la industria del vino y de la cerveza artesanal dan cuenta de ese aprovechamiento de los recursos locales, del aprendizaje colectivo, de la difusión del saber hacer, de la diversificación de actividades a partir del turismo, la vitivinicultura y la gastronomía, y en definitiva, de la capacidad de reinvención de una región con base en la incorporación de nuevas actividades productivas. Sin embargo, tanto para el caso de Ensenada, como para la industria vitivinícola de manera más general, debido a la naturaleza multidimensional de este fenómeno, no se tienen datos suficientes que definan claramente el impacto real de las acciones ligadas al sector vitivinícola en el desarrollo y el bienestar local (Alderete, 2014).

En suma, distintas experiencias dejan ver, por un lado, que para cada contexto va a resultar una respuesta específica, y, por otro, que uno de los mayores desafíos se encuentra en la intersección entre el lugar, la acción empresarial, y la contestación e involucramiento de la población local. Como se examina en los siguientes apartados, el caso de SonoitaElgin y Cananea nos permite estudiar e interpretar estas relaciones.

\section{LAS NUEVAS GEOGRAFÍAS VITIVINÍCOLAS COMO PROTAGONISTAS DE UNA NUEVA FASE DE EXPANSIÓN DE LA INDUSTRIA DEL VINO}

La nueva fase de expansión de la industria del vino aparece ligada al surgimiento de espacios de producción donde la actividad vitivinícola es reciente o emergente (en proceso de experimentación, expansión y desarrollo), y donde la vinculación y conexión con el lugar de producción no se basa directamente en la tradición, la historia, el pasado o la autoctonía del cultivo de la vid, sino en una mezcla de influencias de diversa índole (Banks y Overton, 2010; Paxson, 2010). Algunas de estas resultan de adaptar las características productivas particulares del lugar, por una parte, a los cambios recientes en el mercado del vino y a la demanda específica a distintos niveles (doméstico, nacional e internacional). Por otra, como consecuencia de la adaptación y reinterpretación de ideas hegemónicas en cuanto a la producción y consumo, que provienen del viejo y el nuevo mundo del vino. Todas estas características nos conducen a, identificar, justificar y plantear lo que aquí,

\footnotetext{
${ }^{4}$ Tucson también forma parte de la red de ciudades creativas desde 2015.
} 
8 Nuevas geografías vitivinícolas en la frontera México-Estados Unidos. Un estudio sobre el sentido... Salas Quesada, M.C. y Sandoval Godoy, S.G.

junto con otros autores, llamamos "nuevas geografías vitivinícolas "o "áreas vitivinícolas emergentes" (Banks et al., 2013; Banks y Overton, 2010).

En este trabajo, la idea de las nuevas geografías vitivinícolas responde a la misma lógica propuesta por los estudios regionales con la categoría de "nuevas regiones geográficas" (Paasi, 2002). Se trata de establecer una categoría flexible con la que se busca reinterpretar desde un enfoque crítico "el lugar" (y las identidades asociadas al mismo) de manera contextualizada. Es decir, el lugar no como algo que viene dado sino como un proceso definido "en relación a la experiencia (intersubjetiva) humana localizada" (Tuan, 1975, citado en Paasi, 2002, p. 806). La percepción contextualizada del lugar en el planteamiento de esta categoría coincide, por tanto, con el posicionamiento teórico que sustenta el análisis del sentido del lugar.

Aquí se consideran como NGV países como China, India, Tailandia, Brasil, Perú y México, entre otros; pero también nuevas regiones o microrregiones, que aunque situadas en países con una producción vitivinícola consolidada, hayan sido desarrolladas recientemente.

Hoy en día la industria del vino se ha expandido a distintos niveles (nacional, internacional, regional). Así, dentro de cada país existen regiones y microrregiones vitivinícolas cuyo desarrollo ha ocurrido en condiciones socioeconómicas, políticas, históricas y temporales muy diversas. Como consecuencia, es difícil clasificar y analizar los distintos espacios vitivinícolas de cada país bajo una misma categoría como la de viejo/nuevo/tercer mundo del vino. Esta última categoría, el tercer mundo del vino, se ha asociado a países productores emergentes en la producción de vino con "bajos niveles de desarrollo económico" (Buchanan, 1964; en Banks y Overton, 2010, p. 67). Los dos casos de estudio analizados en este trabajo revelan la imprecisión de este tipo de categorías. Arizona se encuentran dentro del nuevo mundo del vino, sin embargo, su incorporación al sector es muy reciente si la comparamos con otros estados del país, como California. Del mismo modo, Sonora, en México experimenta un desarrollo de la industria vitivinícola más emergente que el de regiones como Baja California, Querétaro, o Coahuila. Por ello, en el presente análisis se realiza un primer acercamiento a estos espacios emergentes desde la noción de NGV.

A pesar de la ausencia de antecedentes productivos, de la escasa representación y de la reducida visibilidad a nivel nacional e internacional, así como del difícil contexto de competencia en el mercado global del vino, las NGV cuentan con una ventaja competitiva, la posibilidad de crecer, de innovar y de experimentar (Banks et al., 2013; Banks y Overton, 2010).

En conjunto, estos nuevos escenarios vitivinícolas rompen, en parte, con el panorama hegemónico clásico del viejo/nuevo mundo del vino. Así, dichos espacios revelan que en el sector vitivinícola global no solo intervienen las regiones protagonistas tradicionalmente conocidas, sino que también entran en escena nuevos actores y lugares de producción antes desconocidos, mismos que han comenzado a descentralizar y resignificar la producción del vino. 


\section{EL TRABAJO DE CAMPO ETNOGRÁFICO}

El posicionamiento teórico (relacional, complejo y exploratorio) explicado en los aparatados anteriores requiere de herramientas analíticas flexibles que permitan estudiar el fenómeno en su contexto de emergencia, experimentación y desarrollo de las NGV. Es por ello que para el análisis de los dos casos seleccionados se utiliza el paradigma cualitativo/crítico, el enfoque interpretativo, y el método etnográfico. Se efectúa un muestreo intencional (Onwuegbuzie y Leech, 2007) con el que se selecciona a informantes claves de acuerdo a su involucramiento y su función (líderes, promotores/difusores, legitimadores, audiencias) dentro del sector vitivinícola (Byrkjeflot, Pedersen y Svejenova, 2013); y a su participación externa o interna en el mismo (Christensen, Kenney y Patton, 2015).

Se seleccionan dos unidades de estudio, la región de Sonoita-Elgin, en Arizona, y Cananea, en Sonora. Se plantea un estudio comparativo por tres razones: la primera, por su pertinencia a la hora de discutir la categoría de NGV (de acuerdo a lo descrito en el apartado anterior). La segunda, por los elementos en común que comparten ambos casos, como se explicará más adelante. Finalmente, en tercer lugar, porque ofrece la posibilidad de realizar una descripción más amplia, un estudio más dinámico y con un alcance mayor sobre el fenómeno observado. Lo anterior, ya que en Cananea solo hay una vinícola establecida, y dos proyectos en preparación; mientras que en Sonoita-Elgin encontramos un total de 15 vinícolas y algunos proyectos más en desarrollo.

Además de la comparación, para este estudio se utilizan la observación y las entrevistas, como técnicas propias del método etnográfico, y otras afines como el análisis del discurso (en prensa y redes sociales). El trabajo de campo fue llevado a cabo de abril de 2018 a marzo de $2020^{5}$ con visitas continuadas a ambos espacios vitivinícolas. En ese periodo se realizaron 32 entrevistas semiestructuradas en español e inglés a diferentes actores claves ${ }^{6}$ involucrados en la producción, en la comercialización y la promoción de esta actividad. Nos centramos en estos actores porque son ellos los que en primera instancia definen, comunican e impulsan la actividad vitivinícola (Boisier, 2009; Byrkjeflot et al., 2013).

Asimismo, las entrevistas se contrastan con la información obtenida de la observación (centrándonos especialmente en la interacción entre productores y distintas audiencias y/o consumidores) en espacios como las salas de degustación en Sonoita-Elgin; en catas organizadas y cenas maridaje en Hermosillo; en las fiestas de la vendimia (2018 y 2019) de

\footnotetext{
${ }^{5}$ El trabajo de campo se interrumpió en marzo de 2020 por el cierre de fronteras y las restricciones de movilidad impuestas por la situación de pandemia por COVID 19.

${ }^{6} \mathrm{La}$ información recopilada de las entrevistas con informantes claves se expone de manera anónima. Lo anterior, por razones éticas y por el derecho de privacidad, confidencialidad y protección de la información compartida en comunicaciones personales entre el entrevistador y los informantes.
} 
10 Nuevas geografías vitivinícolas en la frontera México-Estados Unidos. Un estudio sobre el sentido...

Salas Quesada, M.C. y Sandoval Godoy, S.G.

Cananea; y en distintos festivales como el VinoFest $(2018,2019,2020),{ }^{7}$ en San Carlos, Sonora, México, o el Madero $501,{ }^{8}$ llevado a cabo en Hermosillo, Sonora, entre otros eventos.

Estas son algunas de las principales características del diseño metodológico aplicadas en este trabajo para enfatizar y abordar analíticamente tres aspectos. En primer lugar, la contextualización de los casos, debido a que, como se ha justificado teóricamente, para el análisis del sentido del lugar el contexto es lo básico. En segundo lugar, la interpretación sobre cómo ese contexto condiciona la especificidad de cada espacio vitivinícola. Finalmente, en tercer lugar, la reflexión sobre las diferencias y las posibilidades de interacción entre estas dos áreas transfronterizas.

Aunque todavía en progreso, ${ }^{9}$ se espera que la estrategia metodológica aquí presentada sea útil para investigaciones futuras con las que poder seguir sumando casos en esta misma línea de investigación sobre las NGV y la construcción del sentido del lugar.

\section{CANANEA, SONORA Y SONOITA - ELGIN, ARIZONA COMO NGV: LO ESPECÍFICO, LO COMÚN Y LAS POSIBILIDADES DE INTERACCIÓN}

Las regiones de Cananea, en Sonora, México, y de Sonoita- Elgin, en Arizona, en Estados Unidos, se encuentran localizadas en la frontera norte y sur de ambos países, respectivamente. En Sonoita se puede observar el trabajo de una comunidad y una región que en los años ochenta se aventuró a introducir una función productiva completamente nueva para el territorio, y cuyos resultados son de impacto positivo para su comunidad. Treinta años después, a cien millas de distancia y compartiendo la proximidad a la frontera, Cananea experimenta una experiencia con características similares.

\footnotetext{
${ }^{7}$ Festival organizado desde 2018 por la Comisión de Fomento al Turismo (Cofetur) del estado de Sonora, en la edición de 2019 contó además con la colaboración del Comité Provino de Baja California. En este evento se reúnen a distintos productores de vino nacionales e internacionales y a restauranteros, se realizan conferencias, exposiciones, catas de vino y degustación de la gastronomía regional.

${ }^{8}$ Festival organizado por el Patronato del Parque Madero, Hermosillo, Sonora, Cofradía Centenario HMO, y Salud Fraternal Hermosillo. En la primera edición 2019, y única edición hasta la fecha, se presentaron degustaciones de vino, mezcal, tequila y cerveza artesanal, de productores nacionales e internacionales. En esta ocasión hubo presencia de Callaghan Vineyards, vinícola de Sonoita-Elgin, Arizona, y de la vinícola Uvas de Altura, de Cananea, Sonora.

${ }^{9}$ De acuerdo a la estrategia metodológica, los resultados finales se pretenden discutir bajo las categorías de "especificidad, identificación, representación e interacción", las cuales se integran dentro de la dimensión de territorio y la perspectiva analítica del sentido del lugar. En esa dirección, el presente trabajo supone un primer paso de acercamiento y aproximación a los resultados.
} 


\section{Sonoita-Elgin, Arizona}

En Sonoita la industria vitivinícola re-surge en los años setenta con un proyecto de la Universidad de Arizona dirigido por el Dr. Gordon Dutt, especialista en análisis de suelos. El proyecto tenía el objetivo de encontrar cultivos alternativos con bajo consumo de agua y alto valor añadido. Con el apoyo del gobierno y de la Universidad, se realiza un estudio (Mielke, Dutt, Hughes, Wolfe, Loeffler, Gomez, Bryant, Watson y Schick, 1980) en el que se analiza la viabilidad para este cultivo en diferentes áreas de Arizona, así como en Colorado, Nuevo México y Utah. En 1973, con los primeros resultados positivos de dicho estudio, se establece el primer viñedo experimental en el área de Sonoita. La plantación comienza en el rancho de Babacomari, propiedad de Blake Brophy quien informó al Dr. Dutt de la existencia de unas viñas abandonadas. Las viñas abandonadas eran herencia de las misiones Jesuitas y Franciscanas, y en ellas se encontró la variedad de vitis vinífera conocida con el nombre de misión (Informante \# 1, comunicación personal, 18 de febrero de 2020). En 1974, el Dr. Dutt funda la primera vinícola en el valle de Sonoita-Elgin, Sonoita Vineyards, donde actualmente su nieta, Lori Reynolds, se encarga de la producción.

En 1984 se reconoce al valle de Sonoita-Elgin dentro del sistema de indicaciones geográficas AVA (American Viticultural Areas), ${ }^{10} \mathrm{y}$ desde entonces ha logrado un discreto reconocimiento nacional e internacional y un importante desarrollo a nivel local. Como región vitivinícola se desarrolla especialmente en la década de los 2000, y actualmente cuenta con 15 vinícolas establecidas y algunos proyectos más en planeación.

La aportación de Sonoita-Elgin al mercado nacional e internacional de vino es limitada, ya que en E.E. U.U. 90 por ciento de dicho producto se produce en California (Rendleman, Hoemmen, Altman, Taylor, Moon y Smith, 2016).

En su mayoría, se trata de empresas pequeñas (una media por debajo de 10 hectáreas) y familiares, donde los propietarios están involucrados en todas las tareas, desde el cuidado de la viña y la vinificación, hasta la promoción y las ventas del producto. Uno de los elementos geoclimáticos a destacar de esta zona vitivinícola es su altitud, que corresponde a 1500 metros de altura, y se encuentra entre las sierras de Santa Rita, Huachuca, Whetstone, y Canelo Hills. La altitud, el desfase térmico entre el día y la noche, consecuencia de esta ubicación, y la presencia de monzones de junio a septiembre hacen que esta área reúna unas condiciones singulares para la producción de este cultivo. Con respecto a los tipos de uva, cuentan con una amplia diversidad de variedades entre las que se pueden mencionar las siguientes: sauvignon blanc, cabernet sauvignon, syrah, malbec, petite sirah, tannat, merlot, pinot noir, sangiovese y mission, entre otras.

Por otra parte, una mirada hacia la industria en Sonoita-Elgin (AVA) permite observar un impacto significativo en el desarrollo a nivel de la población local y de la región. Como

${ }^{10}$ El Valle de Sonoita-Elgin fue el primero en incorporar esta actividad, la cual se ha expandido en el estado de Arizona de manera que actualmente son dos las áreas reconocidas como AVA: Sonoita- Elgin, y Willcox. 
12 Nuevas geografías vitivinícolas en la frontera México-Estados Unidos. Un estudio sobre el sentido...

Salas Quesada, M.C. y Sandoval Godoy, S.G.

explica el trabajo de Cubillas, Mars, Torres y Sias (2017), el 95 por ciento del vino producido en esta área es vendido directamente por los productores. Esto se traduce en un efecto positivo en el mercado local, en la economía y en el empoderamiento de estas comunidades rurales. El trato directo con los productores transmite un cierto sentido de comunidad. Como se describe en la publicación Arizona Wine Country, "negocios locales para producir deliciosos productos para la comunidad" (Arizona Wine Country Sonoita \& Elgin, 2018, p. 8 [traducción propia]).

Asimismo, a priori se percibe una producción diferente, o como ellos la definen, "a free spirit production" (The Travel Podcast, 2020). Es decir, que en Sonoita los produtores no se centran en un estilo y una variedad, de acuerdo a los modelos clásicos de producción, sino muchas variedades y muchos estilos. Expresiones como las siguiente dan cuenta de la versatilidad de esta región: "wines from grapes representing all regions of the world" (recuperado del periódico local Arizona Wine Country Sonoita \& Elgin, 2018, p. 8). También se observa cierta originalidad y diversidad en los productos (vino, brandy, licores, cerveza artesanal, sidra) y sus formas de presentación. Encontramos en sus salas de degustación desde la sencillez del paisaje, o los elementos decorativos de la cultura mexicana, hasta productos adaptados a nuevos escenarios de consumo, como el vino rosado espumoso embotellado en lata de la vinícola Dos Cabezas WineWorks, publicitado en redes sociales bajo el ingenioso hashtag "I Pink therefore I can" (Dos Cabezas, 2017).

Lo anterior parece indicar que se trata de un área vitivinícola en la que el emprendimiento local y la creatividad juegan un papel fundamental, y donde la producción ocurre de manera flexible. Es decir, no supeditada a patrones o normas fijas de elaboración, como ocurre en otros países (principalmente en Europa).

\section{Cananea (Sonora, México)}

Las particularidades para el caso de Sonora, por su parte, con menos tiempo y experiencia en el mercado, adquieren hasta hoy un carácter mucho más exploratorio y experimental. La idea de realizar un análisis contrastado de la región de Cananea, Sonora, y Sonoita, Arizona, nos remite inicialmente al informe de reconocimiento que realiza en 2008 la Fundación Sonora para la Innovación, organización que prepara el estudio de evaluación para la plantación vitivinícola en Cananea. En el informe se indica que uno de los factores que hizo replantearse la factibilidad para la producción de vino en Sonora -que por su condición desértica había sido clasificada por estudios anteriores como no cualificada para el cultivo-, era el hecho de que experiencias en otros lugares con características geoclimáticas similares, como Arizona, demostrasen lo contrario. Además, desde el comienzo del proyecto diferentes figuras gubernamentales manifestaron explícitamente el interés de incluir a Arizona en la propuesta de desarrollo turístico y cultural que acompaña al sector vitivinícola:

La ubicación estratégica de Sonora y la alianza con Arizona para consolidar una

mega región económica genera las condiciones ideales para establecer una ruta 
más allá de lo que es el vino y de lo que es la minería (Secretaría de Economía del Gobierno del Estado de Sonora, 2016, párr.1).

Aunque desde hace varias décadas Sonora es el mayor productor de uva de mesa a nivel nacional (Sagarhpa, 2018), Cananea se incorpora en el sector vitivinícola para la producción de vino de manera más reciente, en 2008. El proyecto surge como parte de una iniciativa que comienza con apoyo público $^{11}$ y que posteriormente se logra consolidar como empresa a partir de la inversión privada de un grupo de empresarios de Sonora. Esta iniciativa buscaba originalmente proyectos con los que impulsar el desarrollo económico de la región. Después de haber estudiado la viabilidad para la plantación de vid vinificable en diferentes zonas del estado, la Fundación Sonora encontró que la localidad de Cananea reunía las características geoclimáticas aptas para dicho cultivo e identifican que existe un potencial en la región como nuevo polo vitivinícola (Susarrey y Moreno, 2010).

Así, el proyecto inicia con una plantación experimental de 30 variedades de vid en 27 hectáreas en el ejido Zaragoza, a las afueras del municipio de Cananea, en dirección al pueblo fronterizo de Naco. Se seleccionaron las variedades que tuvieron mejor adaptación, entre ellas: malbec grenache, carignan, syrah, touriga nacional, petit verdot, carmenere, tempranillo, cabernet sauvignon, mourvedre, chardonnay y verdejo. La empresa encargada tanto del cultivo como de la producción de vino toma el nombre de Uvas de Altura, y sus vinos se denominan Cuatro Sierras, haciendo alusión -al igual que ocurre en Sonoita (Arizona) - a la altitud de la zona, situada a más de 1400 metros, y a su ubicación montañosa entre las sierras de Sierra Vista (Huachuca, Arizona), San José, Los ajos y La Mariquita.

La primera vendimia comercial se realizó en 2015, y a pesar de la incipiente incorporación de la empresa Uvas de Altura en el sector vitivinícola a nivel regional ${ }^{12}$ y nacional, la empresa ha logrado impulsar el proyecto de manera exitosa. Lo anterior queda demostrado por su rápido crecimiento en producción y ventas. Los datos recopilados en las entrevistas señalan que en 2015 se produjeron 28000 botellas, y para 2019 su producción se había triplicado. La producción se comercializa principalmente con la etiqueta de Cuatro Sierras, pero también se destina una pequeña parte al comercio a granel y a la distribución nacional con marcas adicionales elaboradas para conocidas cadenas de supermercados. Con todo esto, según sus promotores, se espera que la actividad vitivinícola pueda seguir creciendo y expandirse hacia los municipios de Naco y Santa Cruz, Sonora, zonas con condiciones geográficas similares y con mayor disponibilidad de agua. De hecho, el agua

${ }^{11}$ En las primeras fases de evaluación y validación del proyecto a través de fondos concursables tanto del Consejo Nacional de Ciencia y Tencnología (Conacyt) de México, como de la Secretaría de Economía del estado de Sonora; y posteriormente, con apoyo estatal y/o municipal para la realización de las fiestas de la vendimia en Cananea, y en otras iniciativas como la construcción de la Casa del Vino (espacio para la organización de eventos y degustaciones).

${ }^{12}$ Existe también un pequeño viñedo en el municipio de Ures, Vinos Giottonini, proyecto iniciado de manera personal por Francisco Salazar Giottonini. 
14 Nuevas geografías vitivinícolas en la frontera México-Estados Unidos. Un estudio sobre el sentido...

Salas Quesada, M.C. y Sandoval Godoy, S.G.

es un recurso limitante en Cananea, ya que en esta localidad se ubica la mina Buena Vista del Cobre, una de las más grandes del mundo, con altos requerimientos de este recurso.

Cabe mencionar también la repercusión de la iniciativa vitivinícola con respecto al desarrollo de las comunidades donde se implanta. De acuerdo a nuestros informantes del equipo de producción, la empresa Uvas de Altura ha generado alrededor de 20 empleos permanentes y hasta 160 empleos temporales. En este sentido, el impulso de la vitivinicultura supone una alternativa a la industria minera, principal actividad económica en Cananea; una fuente de empleo para las áreas rurales ejidales donde se encuentra el viñedo, así como para otras localidades cercanas.

Entre los potenciales y los planes a futuro que presentaron inicialmente los impulsores del proyecto en Cananea, se planteó además la idea sobre la creación de una ruta del vino binacional Sonora - Arizona. En las siguientes citas se comprueba cómo desde el discurso político de distintos actores que apoyan la iniciativa vitivinícola se transmite la idea de crear dicha ruta del vino binacional. Lo anterior, con la intención de promover el desarrollo regional en diferentes ámbitos:

Se tiene proyectado crear una zona vinícola como la de Guadalupe o Apple Valley con todo el aspecto turístico y contar con una ruta internacional del vino al estar en contacto con los productores de Arizona (Dossier Político, 2014a, párr.6). Con este proyecto arranca lo que en el futuro será la Ruta del Vino en SonoraArizona buscando potenciar esta zona como un gran atractivo para los conocedores del vino (Dossier Político, 2014b, párr. 9).

La iniciativa de crear una ruta binacional del vino ha cobrado interés en el ámbito gubernamental y ha sido sumada a uno de los objetivos estratégicos concebido hace poco más de dos décadas por la Comisión Sonora-Arizona; esto es, el de construir una mega región económica entre ambos países. Como parte de esta alianza transfronteriza, en este tipo de propuestas se ha manifestado también la posibilidad de colaborar con ciudades como Sierra Vista y Bisbee (ambas en el estado de Arizona). Sin embargo, a pesar del interés manifestado por distintos actores políticos de la región de Sonora, actualmente no se tienen datos que demuestren que se esté llevando a cabo alguna acción clara y articulada binacionalmente. Lo anterior se justifica si tenemos en cuenta que la producción vitivinícola en Cananea se encuentra aún en una fase incipiente, por lo que este tipo de propuestas parece presentarse como un proyecto a futuro.

Así pues, atendiendo las características expuestas en los casos estudiados, encontramos que esta condición de frontera en colindancia, reúne en estas dos NGV una serie de puntos en común y ventajas comparativas y competitivas fundamentales como son: la proximidad, ya que para Cananea la región vitivinícola más cercana es la región de Sonoita-Elgin, en Arizona, de la cual la separan alrededor de 100 millas de distancia; la innovación, ser pioneros en introducir la vitivinicultura en regiones donde ha predominado fundamentalmente la actividad minera y ganadera; la visibilidad, si bien ambos lugares se consideran secundarios o periféricos para el sector del vino a escala global, a nivel local suponen una nueva imagen y una revitalización del espacio rural; el desarrollo, en tanto 
que la producción de vino aparece como alternativa para el empoderamiento y crecimiento de las comunidades; la flexibilidad, ya que al menos hasta el momento tanto en Cananea como en Sonoita la producción se lleva a cabo en base a diferentes variedades y distintos estilos; y finalmente, la posibilidad de colaboración binacional en proyectos de carácter económico, turístico y sociocultural.

Estos elementos podrían estar caracterizando y estructurando parte de algunos resultados que, aunque incipientes, parecen estar a la vista.

\section{DEL DISCURSO A LA PRÁCTICA: DIFERENCIAS Y CONDICIONAMIENTOS DE LA PRODUCCIÓN VITIVINÍCOLA EN SONORA-ARIZONA}

Los proyectos asociados a la producción sociocultural de las actividades que dan soporte a la producción del vino van acompañados de un discurso impulsado principalmente por los actores políticos. El discurso tiene como objetivo animar a los individuos de la localidad y la región a formar parte de este propósito cultural y económico. Así, discursos, como el que se señalaba a propósito de la creación de una ruta del vino binacional Sonora-Arizona, se apoyan en parte en la reproducción de un imaginario que tiene como función "inventar y proyectar futuros posibles" (Giménez, 2007, p. 250); en ocasiones, distantes de las posibilidades y la práctica del contexto real.

En este sentido, se observa que como indica Ingold (1993), frente al proceso a menudo se privilegia la interpretación del producto final de una determinada actividad. Por eso, la reflexión aquí presentada ha insistido en la importancia del análisis del sentido y la producción del lugar. Con ello se busca una comprensión acabada del espacio-tiempo para cada proyecto, de tal manera que se puedan describir e interpretar las particularidades del contexto que lo produce y lo hace posible.

Así, cuando se señala la dependencia y el condicionamiento que tiene cada iniciativa para un contexto determinado, nos estamos refiriendo por una parte a la dependencia de unos recursos naturales y socioeconómicos. Por otra, a una serie de condicionamientos que vienen dados del espacio- tiempo específico en el cual los creadores (productores de vino y demás actores involucrados en el sector) se posicionan con respecto a: una época dada; un código; una relación con la sociedad, con los consumidores, con sus gustos y con sus demandas (Bourdieu, 1998). En consecuencia, la respuesta y el posicionamiento tanto de productores como de consumidores ante esa situación particular va a condicionar el resultado. De este modo, si anteriormente se apuntaban los aspectos en común, a continuación se analizarán algunas de las diferencias y particularidades para cada caso.

Con base en la información recabada en el trabajo de campo, en Cananea encontramos las siguientes características generales: un proyecto que nace de la asociación de un conjunto de empresarios sonorenses; una extensión de 25 hectáreas, con la intención de expandirse; un estilo de producción que inicialmente privilegia los vinos tintos con maduración en barrica, y al que actualmente se ha incorporado una nueva línea de vinos 
16 Nuevas geografías vitivinícolas en la frontera México-Estados Unidos. Un estudio sobre el sentido...

Salas Quesada, M.C. y Sandoval Godoy, S.G.

jóvenes (blancos y tintos); un rango de precios entre los 250 y 550 pesos; y una distribución a nivel local, estatal y nacional creciendo progresivamente.

Aunque todavía impreciso, este perfil parece tener sentido si lo comparamos con los datos que rescatamos del estudio que realiza el Instituto de Comercio Exterior (ICEX) de España sobre exportación e inversiones ${ }^{13}$ en el que se analiza el mercado del vino en México. El informe señala como una característica importante que se trata de un sector donde la producción nacional ha venido experimentando un rápido crecimiento en los últimos años. Este rápido crecimiento responde a una oportunidad de mercado, debido a que el vino nacional solo cubre alrededor de 30 por ciento de la demanda, y al aumento en el consumo de esta bebida. A pesar de este aumento, el vino en México es un producto todavía poco consumido a nivel doméstico. Entre los vinos más comprados se encuentran los tintos $-61.4 \%$ (Amo, 2018, p. 8)- con un precio en torno a los 200 pesos, segmento medio de precios en el que se ubican "principalmente marcas importadas, con la excepción de algunas marcas mexicanas como Domecq y La Cetto (sic)" (Amo, 2018, p. 28).

De estos datos se puede deducir que el vino hecho en México, aunque con precios muy variables, sigue siendo inaccesible para buena parte de la población mexicana, lo cual limita su inclusión en el consumo doméstico. No obstante, este mismo informe apunta que los patrones de producción y consumo en México están cambiando debido a la incorporación "de un segmento más joven y dinámico de la población [...] y del público femenino" (Amo, 2018, p. 6).

Al menos para el caso de Sonora, esta información se confirma a través de la observación participante en diferentes eventos de vino. En ellos, si bien puede reconocerse el creciente interés de integrar a este "sector joven y dinámico" de la población, todavía predomina la idea de exclusividad y la presencia de más hombres que mujeres, y más adultos que jóvenes. Cabe señalar que México tiene una población joven muy alta, con una clase media que está creciendo, por lo que quizás cabría esperar-como concluye el estudio de ICEX- "la introducción de vinos de precios asequibles a todos los bolsillos, con una excelente relación calidad-precio" (Girón, 2014, p. 28).

Lo anterior no impediría que la misma vinícola pueda tener una línea de vinos con precios más elevados, algo común en esta industria. Covarrubias y Thach (2015) sugieren que esta oportunidad de negocio requiere una acción dirigida a acercar el vino al público mexicano, y aprovechar que el "saludable orgullo y creciente interés por los productos locales puede ser de gran ayuda para la industria del vino en México" (2015, p. 114 [traducción propia]). La campaña nacional lanzada en 2018 titulada Todos unidos por el vino mexicano (Reyes, 2018), con objetivos como el que persigue el Consejo Mexicano Vitivinícola, que para 2028 cinco de cada 10 botellas consumidas en el país sean de vino mexicano, demuestra que existe conciencia de esta limitación, que se está trabajando en

${ }^{13}$ Se han revisado los datos correspondientes con el informe de 2014 y 2018. 
impulsar el consumo local y, a nivel más amplio, que el panorama vitivinícola mexicano está cambiando.

Por su parte, en Sonoita-Elgin se ubica una producción local, flexible y creativa, que ha sido resultado de proyectos que en su mayoría son familiares. Las vinícolas presentan un tamaño medio de entre 4 y 8 hectáreas, un perfil de consumidores heterogéneo, una producción también heterogénea, y un modelo de ventas local. Este modelo de negocio, pequeño, local, familiar y accesible, hace posible el trato directo entre productores y consumidores, relación que se convierte en una característica fundamental de esta región vitivinícola.

El trabajo de Cubillas et al. (2017), analiza la industria vitivinícola en la región de Sonoita-Elgin desde el concepto de co-creación del valor. El estudio revela que dicho modelo hace que los turistas y el público en general describan su experiencia en esta área como "con los pies en la tierra, accesible y honesta" (Cubillas et al., 2017, p. 42 [traducción propia]). Esa descripción de la experiencia coincide ampliamente con lo expuesto por uno de nuestros informantes clave, productor de vino en Sonoita - Elgin, cuando se le preguntó acerca de “¿cuál era el secreto?” del éxito y la buena aceptación del público en esta área vitivinícola, a lo que responde:

Creo que el secreto es buen vino y buen ambiente. Nadie aquí es pretencioso, todos somos personas con los pies en la tierra, somos agricultores, esta es la manera en que producimos, les guste o no. Y a la gente le gusta eso, les gusta venir, les gusta hablar con los dueños, y en la mayoría de las veces somos nosotros los que estamos detrás de la barra, creo que eso es lo que le gusta a la gente y con lo que se quedan, es como estar en casa, les invitamos a nuestra casa a beber con nosotros (Informante \# 1, comunicación personal, 18 de febrero de 2020).

El compartir el conocimiento y la experiencia de manera directa contribuye a que se produzca una "desmitificación” de este producto y "desenmascara la sofisticación del consumo de vino" (Cubillas et al., 2017, p. 48 [traducción propia]). Además, esta desmitificación se transmite por la forma en la que se presentan las vinícolas y sus productos.

En definitiva, se observa que, de manera general, esta zona vititivinícola rompe con el estilo y el código conservador y, en ocasiones, clasista (Bourdieu, 2010) que a menudo encontramos en otras áreas o entornos de producción y consumo de vino. Sin embargo, de la observación y las entrevistas, también se deduce que los propietarios están abiertos a la posibilidad de crecer; que cada vinícola tiene una personalidad diferente; y que no todos los productores están de acuerdo acerca de buscar un mismo modelo de producción y negocio. Es decir que, como se mencionaba al inicio, se percibe una aparente sensación de comunidad entre productores y consumidores, y en consecuencia, una conexión entre el producto, el lugar y la población local. No obstante, también es evidente que en el proceso de construcción y desarrollo de esta NGV existen diferencias, desacuerdos, problemas y conflictos internos. 
18 Nuevas geografías vitivinícolas en la frontera México-Estados Unidos. Un estudio sobre el sentido... Salas Quesada, M.C. y Sandoval Godoy, S.G.

Finalmente, conviene aclarar que para ambos casos, en primer lugar, se trata de una caracterización general que forma parte de los primeros resultados de investigación, que requieren concretarse en posteriores trabajos. En segundo, que refleja únicamente lo que observamos en el presente (conscientes del desfase temporal que existe en el origen de esta actividad entre ambos lugares), lo cual no significa que estas dinámicas vayan a permanecer inamovibles. Es decir, que la interacción espacio-tiempo es cambiante, especialmente si consideramos que se trata de NGV en fase de experimentación, crecimiento y desarrollo.

Lo que se pretende evidenciar con este análisis comparativo es que dos espacios con características geoclimáticas similares (y otros puntos en común anteriormente explicados), pueden dar lugar a dos regiones vitivinícolas con perfiles diferentes con respecto a la producción y el consumo. Con ello se establecen las premisas para comprobar lo que se señalaba al inicio de este trabajo: 1) que la variabilidad de cada región vitivinícola depende de un proceso de producción del lugar, en el que, como se ha mostrado, los intereses de diferentes actores y el posicionamiento de productores y consumidores con respecto a una época dada son determinantes; 2) que la intersección entre el lugar, la acción empresarial, y la contestación e involucramiento de la población local es fundamental en el proceso de diferenciación, diversificación y desarrollo de esta actividad, y en consecuencia, 3) que para cada contexto va a resultar una respuesta específica.

Con todo y las coincidencias y divergencias observadas, Cananea y Sonoita-Elgin han conseguido de manera independiente y exitosa introducir una nueva función productiva y romper con el escepticismo (nacional e internacional) acerca de que en Sonora y Arizona se puede producir vino y demostrar su calidad. En ese sentido, una de nuestros informantes, productor de vino en Sonoita-Elgin, señaba la desconfianza inicial a la que se enfrentaron los precursores de la iniciativa vitivinícola en el estado de Arizona: "Mucha gente lo miraba como si estuviese loco porque, ya sabes, es Arizona, la gente piensa en cactus, vaqueros, y serpientes de cascabel" (Informante \# 1, comunicación personal, 18 de febrero de 2020). Ese mismo escepticismo se observa para el caso de Sonora.

La incorporación de la industria vitivinícola en Sonora y Arizona ha desencadenado también una serie de efectos positivos para las localidades donde se ubican. Al mismo tiempo, estas nuevas geografías vitivinícolas incorporan nuevos actores en el sector del vino a escala global. Por lo tanto, las diferencias encontradas no se interpretan como negativas. Al contrario, sugieren que para poner en práctica una colaboración, hay que pasar de lo efímero, de una idea y un discurso, a lo material; esto es, un diálogo entre productores y un intercambio de conocimiento. Para ello, en lo sucesivo, se habrá de reflexionar en la conveniencia de aprovechar la cercanía y los puntos en común de estas dos áreas e impulsar (micro) interacciones con las que establecer las bases para futuros (macro) proyectos, como puede ser una ruta binacional. En este sentido, el mayor potencial con que cuentan las regiones fronterizas es que ante todo se trata de espacios relacionales 
(Peña, 2017) en los que poder compartir el dialogo, y contrastar las ideas, los valores y las prácticas en torno, en este caso, a la producción vitivinícola.

\section{CONCLUSIONES}

Como se ha podido observar en este trabajo, la reciente expansión y crecimiento de la industria del vino a nivel mundial ha ocasionado el surgimiento de $\mathrm{NGV}$ en las que se están formulando respuestas locales muy diversas. De tal forma, de acuerdo con Banks y Overton (2010) y Banks et al., (2013), no podemos analizar el desarrollo global de la industria vitivinícola como una actividad homogénea que depende únicamente de una adaptación geográfica. Contrariamente, lo que se aprecia es un desarrollo profundamente diferenciado, donde el contexto y la interacción en el mismo entre los actores/la actividad/la población local se convierte en uno de los aspectos determinantes en la configuración de la especificidad de cada región vitivinícola.

Cananea, Sonora y Sonoita-Elgin, Arizona, como unidades de estudio representativas dentro de la categoría más amplia de $\mathrm{NGV}$, resultan relevantes ya que contribuyen a elaborar interpretaciones alternativas sobre el proceso de creación del sentido del lugar. Es decir que, como ha señalado Massey (1991), se trata de experiencias que sugieren no caer en el error de pensar que la identidad y el sentido del lugar es una construcción únicamente del viejo mundo y de años de tradición, sino que existe la posibilidad de proponer versiones alternativas e identidades múltiples acordes a espacios heterogéneos, como lo es el espacio transfronterizo. Ciertamente, la conexión entre la vitivinicultura, las nuevas geografías y el proceso de creación del sentido del lugar ofrecen la oportunidad de crear algo innovador, de reinventar el territorio, de activar procesos de reterritorialización (Flores, 2007), de (re)localizarse y de volver a dar visibilidad a una región (Urry, 1995). Sin embargo, como mencionábamos al inicio, el que exista esa potencialidad es solo el primer paso (Appadurai, 1996). En su fase de emergencia y desarrollo, Cananea y Sonoita han comenzado a activar este tipo de fenómenos, aunque con los datos recopilados hasta ahora no podemos adelantar los efectos de la materialización de los mismos ni su repercusión real en el desarrollo local.

Además, ambos espacios vitivinícolas se sitúan como nuevas geografías vitivinícolas ubicadas en una intersección entre lo global y lo regional, pero también lo transnacional y lo fronterizo. Este escenario nos habla no sólo de compartir una misma geografía y una actividad productiva, sino que también ofrece de manera indirecta un espacio donde examinar las posibilidades de interacción entre ambos lugares. Avanzar en esa dirección habrá de requerir del diseño de futuras líneas de análisis que promuevan las redes de comunicación (Lara, 2017), de aprendizaje, de creatividad, de innovación (Amin, 2008), y de aprovechamiento inteligente de las asimetrías (Wong, 2005). Asimismo, de estudios interculturales con los que se pueda reconocer con mayor profundidad lo diferente, lo 
20 Nuevas geografías vitivinícolas en la frontera México-Estados Unidos. Un estudio sobre el sentido...

Salas Quesada, M.C. y Sandoval Godoy, S.G.

antagónico, lo común y lo específico (Giménez, 2010) de la industria vitivinícola para ambos espacios.

Aquí nos hemos ocupado de estudiar el vino y la vitivinicultura como un producto mercantil y cultural, remarcando la importancia de prestar atención a las particularidades socio-culturales y contextuales de cada localidad. Aun con sus limitaciones, bajo este enfoque es posible ofrecer explicaciones quizá más integradas y menos parciales acerca de quiénes somos como región y sociedad, y cómo nos diferenciamos y valorizamos; preguntas que por demás constituyen y han constituido temas de análisis central en las interpretaciones recientes sobre el cambio social.

\section{REFERENCIAS}

Alderete, M. V. (2014). The Wine Clusters of Mendoza and Serra Gaúcha: A Local Development Perspective. Frontera Norte, 26(52), 179-203.

Amin, A. (2008). La política regional en una economía global. En V.R. Fernández, A. Amin y J.I. Vigil (Comps.), Repensando el Desarrollo Regional. Contribuciones globales para una estrategia latinoamericana, (pp. 354-372). Buenos Aires: Miño y Dávila.

Amo, J. (2018). El mercado del vino en México. México: ICEX España Exportación Inversiones. Recuperado de http://www.ivace.es/Internacional_InformesPublicaciones/Países/México/Mexicovinoicex2018.pdf

Appadurai, A. (1986). Introduction: commodities and the politics of value. In A. Appadurai (Ed.), The Social Life of Things, (pp. 3-63). Reino Unido: Cambridge University Press.

Appadurai, A. (1996). Global Ethnoscapes: Notes and queries for a transnational anthropology. En A. Appadurai (Ed.), Modernity at large: Cultural dimensions of globalization, (pp. 48-65). Mineápolis: University of Minnesota Press.

Arizona Wine Country Sonoita \& Elgin. (abril de 2018). Recuperado de https://issuu.com/wickcommunications/docs/wine_country_2018

Banks, G., Klinsrisuk, R., Dilokwanich, S. y Stupples, P. (2013). Wines without Latitude: Global and local forces and the geography of the Thai wine industry. EchoGéo, 23, 018.

Banks, G. y Overton, J. (2010). Old world, new world, third world? Reconceptualising the worlds of wine. Journal of Wine Research, 21(1), 57-75.

Barham, E. (2003). Translating terroir: The global challenge of French AOC labeling. Journal of Rural Studies, 19(1), 127-138.

Boisier, S. (2009). El Retorno Del Actor Territorial a Su Nuevo Escenario. Ambienta: La revista del Ministerio de Medio Ambiente, (89), 112-141. 
Bourdieu, P. (1998). La distinción: Criterios y bases sociales del gusto [Trad. M. C. Ruiz]. Madrid: Taurus.

Bourdieu, P. (2010). El Sentido Social Del Gusto. Elementos para una sociología de la cultura [Trad. A. Gutiérrez]. Buenos Aires: Siglo XXI Editores

Bowen, S. (2010). Embedding local places in global spaces: Geographical indications as a territorial development strategy. Rural Sociology, 75(2), 209-243.

Byrkjeflot, H., Pedersen, J. S. y Svejenova, S. (2013). From label to practice: The process of creating new Nordic cuisine. Journal of Culinary Science and Technology, 11(1), 3655.

Christensen, B. C., Kenney, M. y Patton, D. (2015). Regional identity can add value to agricultural products. California Agriculture, 69(2), 85-91.

Contreras, H. J. y Gracia, A. M. (2005). Alimentación y cultura: Perspectivas antropológicas. Barcelona, España: Ariel.

Covarrubias, J. y Thach, L. (2015). Wines of Baja Mexico: A qualitative study examining viticulture, enology, and marketing practices. Wine Economics and Policy, 4(2), 110115 .

Cubillas, S. Matthew M. M., Torres, R.M. y Sias P.M. (2017). Touristic Authenticity and Value Co- Creation: An Exploration of Two Local Wineries in Southeastern Arizona, USA. Journal of Rural and Community Development, 12(1), 34-54.

De Marchi, V. y Grandinetti, R. (2016). Industrial districts evolving in glocal value chains: evidence from the Italian wine industry. Piccola Impresa/Small Business, (1), 10-36.

Demossier, M. (2013). Following grands crus: Global markets, transnational histories and wine. En R. Black y R. C. Ulin (Eds.), Wine and Culture: Vineyard to Glass, (pp. 183200). Londres: Bloomsbury.

Demossier, M. (2018). Burgundy: The global story of terroir. Nueva York: Berghahn Books.

Dos Cabezas. (2 de diciembre de 2017). Fall afternoon picnic on the dam. \#ipinkthereforeican. [imagen de Instagram]. Recuperado de https://www.instagram.com/p/BcN_aplfhG/

Dossier Político. (29 de abril de 2014a). Cananea producirá vinos como los franceses. Autor. Recuperado de https://www.dossierpolitico.com/vernoticiasanteriores.php?artid=142503\&relacion=dos $\underline{\text { sierpolitico \& criterio }=0}$

Dossier Político. (1 de septiembre de 2014b). Coloca Padrés primera piedra de la Casa del Vino en Cananear Autor. Recuperado de https://www.dossierpolitico.com/vernoticiasanteriores.php?artid=147907\&relacion=dos 
22 Nuevas geografías vitivinícolas en la frontera México-Estados Unidos. Un estudio sobre el sentido...

Salas Quesada, M.C. y Sandoval Godoy, S.G.

\section{$\underline{\text { sierpolitico\&criterio }=0}$.}

Escobar, A. (2001). Culture sits in places: Reflections on globalism and subaltern strategies of localization. Political Geography, 20(2), 139-174.

Ferguson, P. (1998). A cultural field in the making: Gastronomy in nineteenth-century France. American Journal of Sociology, 104(3), 597-641.

Fernández, M. J., Vaillant, Y., Lafuente, E. y Moreno, J. (2017). The renaissance of a local wine industry: The relevance of social capital for business innovation in DOQ El Priorat, Catalonia. Wine Economics and Policy, 6(2), 136-145.

Flores, M. (2007). La identidad cultural del territorio como base de una estrategia de desarrollo sostenible. Opera, (7), 35-54.

Friedman, J. (1994). Identidad cultural y proceso global [Trad. E. Sinnott]. Buenos Aires: Amorrortu Editores

Giménez, G. (2007). Estudios sobre la cultura y las identidades sociales. México: Consejo Nacional para la Cultura y las Artes/Instituto Coahulense de Cultura.

Giménez, C. (2010). El Interculturalismo: Propuesta Conceptual y Aplicaciones Prácticas. España: Cuadernos Ikuspegi/Observatorio Vasco de Inmigración

Giménez, G. (1997). Materiales para una teoría de las identidades sociales. Frontera Norte, 9(18), 9-28.

Giménez, G. (1999). Territorio, cultura e identidades, la región socio-cultural. Época II, $V(9), 25-57$.

Girón, P. (2014). El mercado del vino en México. España: ICEX, España Exportación e Inversiones. Recuperado de http://www.ivace.es/Internacional_InformesPublicaciones/Países/México/Mexicovinoicex2018.pdf

Haesbaert, R. (2011). El mito de la desterritorialización: del fin de los territorios a la multiterritorialidad. México: Siglo XXI Editores

Harvey, D. (2002). The art of rent: globalization, monopoly and commodification of culture. Socialist Register, (38), 93-110.

Herzfeld, M. (2004). The body impolitic. Artisans and artifice in the global hierarchy of value. Chicago: The University of Chicago Press.

Hillel, D., Belhassen, Y. y Shani, A. (2013). What makes a gastronomic destination attractive? Evidence from the Israeli Negev. Tourism Management, 36(C), 200-209.

Hira, A. y Swartz, T. (2014). What makes Napa Napa? the roots of success in the wine industry. Wine Economics and Policy, 3(1), 37-53.

Ingold, T. (1993). The Temporality of the Landscape Revisited. World Archaeology, 25(2), $152-174$. 
Jung, Y. (2014). Tasting and judging the unknown terroir of the Bulgarian wine: The political economy of sensory experience. Food and Foodways, 22, 24-47.

Lara, F. (2017). Estructuras y comunidades transfronterizas: un enfoque de redes sociales de la gestión del desarrollo en la frontera México-Estados Unidos. En M. R. Barajas Escamilla, E. J. Varela Álvarez y P. Wong González (Coords.), Entre fronteras: Construyendo una agenda global comparada, (pp. 5-76). México: El Colegio de la Frontera Norte/Centro de Investigación en Alimentación y Desarrollo.

Massey, D. (1991). A Global Sense of Place. Marxism Today, (411), 24-29. Recuperado de http://banmarchive.org.uk/collections/mt/pdf/91_06_24.pdf

Moran, W. (1993). The Wine Appellation as Territory in France and California. Annals of the Association of American Geographers, 83(4), 694-717.

Mielke, E., Dutt, G., Hughes, S., Wolfe, W., Loeffler, G., Gomez, R., Bryant, M., Watson, J. y Schick, S. (1980). Grape and Wine Production in the Four Corners Region. E.E. U.U.: College of Agriculture/University of Arizona. Recuperado de http://hdl.handle.net/10150/602124

Murdoch, J., Marsden, T. y Banks, J. (2000). Quality, nature and embeddedness: Some theoretical considerations in the context of the food sector. Economic Geography, 76(2), 107-125.

Onwuegbuzie, A. J. y Leech, N. L. (2007). A call for qualitative power analyses. Quality \& Quantity: International Journal of Methodology, 41(1), 105-121.

Paasi, A. (2002). Place and region: Regional worlds and words. Progress in Human Geography, 26(6), 802-811.

Paxson, H. (2010). Locating Value in Artisan Cheese: Reverse Engineering Terroir for New-World Landscapes. American Anthropologist, 112(3), 444-457.

Peña, S. (2017). Perspectivas teóricas sobre la(s) frontera(s). En M. R. Barajas escamilla, E.J. Varela Álvarez y P. Wong González (Coords.), Entre fronteras: Construyendo una agenda global comparada, (pp. 23-38). México: El Colegio de la Frontera Norte/Centro de Investigación en Alimentación y Desarrollo.

Prats, L. (1997). Antropología y patrimonio. Colección Ariel Antropología. Barcelona: Editorial Ariel.

Raftery, D. (2017). Producing value from Australia's vineyards: an ethnographic approach to "the quality turn" in the Australian wine industry. Journal of Political Ecology, 24(1), 342.

Rainer, G. (2016). The making of the, world's highest wine region': Globalization and viticulture restructuring in Salta (NW-Argentina). Erdkunde: archive for scientific geography, 70(3), 255-269. 
24 Nuevas geografías vitivinícolas en la frontera México-Estados Unidos. Un estudio sobre el sentido...

Salas Quesada, M.C. y Sandoval Godoy, S.G.

Rendleman, C., Hoemmen, G. A., Altman, I., Taylor, B., Moon, W. y Smith, S. (2016). Wine Industry Competitiveness: A survey of the Shawnee Hills American Viticultural Area. Wine Economics and Policy, 5(1), 4-13.

Reyes, C. I. (14 de mayo de 2018). Ponen en marcha la campaña "Todos Unidos por el Vino Mexicano". Enfoque noticias. Recuperado de https://enfoquenoticias.com.mx/noticias/ponen-en-marcha-la-campa-todos-unidos-porel-vino-

mexicano?_cf_chl_jschl_tk_=pmd_3_28LYZyngG0WspzfRmloL8QvKV7IcNUe2pZ 8X7XsVo-1632249971-0-gqNtZGzNAiWjenBszQj1

Secretaría de Agricultura, Ganadería, Recursos Hidráulicos, Pesca y Acuacultura (Sagarhpa). (2018). ¿Sabias qué? Sonora es líder en la producción nacional de uva. México: Gobierno de Sonora. Recuperado de http://oiapes.sagarhpa.sonora.gob.mx/notas/econo/uva.pdf

Secretaría de Economía del Gobierno del Estado de Sonora. (3 de septiembre de 2016). Inauguración del primer Festival Cultural de la Vendimia. Autor. Recuperado de https://www.economiasonora.gob.mx/portal/prensa/noticias/213-vino-cananea-uvafestival

Schmidt, C. M., Macchione, M. S. y Fowler de Ávila, G. (2014). Value Creation and Value Appropriation in Networks: an Empirical Analysis of the Role of Geographical Indication in the Wine Industry in Vale Dos Vinhedos, Rs, Brazil. Organizações Rurais \& Agroindustriais, 16(3), 343-362.

Susarrey, J. G. y Moreno, C. (2010). Estudio climatológico de la región de Cananea para una plantación experimental de uva para vino. En Seminario de Vitivinicultura 2010, (pp. 47-53). México: Secretaría de Agricultura, Ganadería, Desarrollo Rural, Pesca y Alimentación/Instituto Nacional de Investigaciones Forestales, Agrícolas y Pecuarias/Centro de Investigación Regional del Noroeste/Campo Experimental Costa de Hermosillo. Recuperado de https://docplayer.es/65273951-Seminario-de-viticultura2010.html

Swyngedouw, E. (1999). Modernity and hybridity: Nature, regeneracionismo, and the production of the Spanish waterscape, 1890-1930. Annals of the Association of American Geographers, 89(3), 443-465.

The Travel Podcast. (24 de agosto de 2020). Arizona Wine Region. Free spirited wine makers and the destination that inspires them! [podcast]. Recuperado de https://www.thetravelpodcast.com/arizona-wine-region/

Trubek, A. B. y Bowen, S. (2008). Creating the taste of place in the United States: Can we learn from the French? GeoJournal, 73(1), 23-30.

Urry, J. (1995). Consuming places. Londres: Routledge, Taylor \& Francis Group. 
Winter, M. (2003). Embeddedness, the new food economy and defensive localism. Journal of Rural Studies, 19(1), 23-32.

Wong, P. (2005). La Emergencia de Regiones Asociativas Transfronterizas: Cooperación y Conflicto en la Región Sonora-Arizona. Frontera Norte, 17(33), 77-106.

Zhao, W. (2005). Understanding classifications: Empirical evidence from the American and French wine industries. Poetics: Journal of empirical research on literature, the media and the arts, 33(3), 179-200. 